\title{
Phase transition and landscape statistics of the number partitioning problem
}

\author{
Peter F. Stadler, ${ }^{1,2,3}$ Wim Hordijk, ${ }^{4}$ and José F. Fontanari ${ }^{4}$ \\ ${ }^{1}$ Bioinformatik, Institut für Informatik. Universität Leipzig, Kreuzstraße 7b, D-04103 Leipzig, Germany \\ ${ }^{2}$ Institut für Theoretische Chemie und Molekulare Strukturbiologie, \\ Universität Wien, Währingerstraße 17, A-1090 Wien, Austria \\ ${ }^{3}$ The Santa Fe Institute, 1399 Hyde Park Road, Santa Fe, NM 87501, USA \\ ${ }^{4}$ Instituto de Física de São Carlos, Universidade de São Paulo, \\ Caixa Postal 369, 13560-970 São Carlos SP, Brazil.
}

\begin{abstract}
The phase transition in the number partitioning problem (NPP), i.e., the transition from a region in the space of control parameters in which almost all instances have many solutions to a region in which almost all instances have no solution, is investigated by examining the energy landscape of this classic optimization problem. This is achieved by coding the information about the minimum energy paths connecting pairs of minima into a tree structure, termed a barrier tree, the leaves and internal nodes of which represent, respectively, the minima and the lowest energy saddles connecting those minima. Here we apply several measures of shape (balance and symmetry) as well as of branch lengths (barrier heights) to the barrier trees that result from the landscape of the NPP, aiming at identifying traces of the easy/hard transition. We find that it is not possible to tell the easy regime from the hard one by visual inspection of the trees or by measuring the barrier heights. Only the difficulty measure, given by the maximum value of the ratio between the barrier height and the energy surplus of local minima, succeeded in detecting traces of the phase transition in the tree. In adddition, we show that the barrier trees associated with the NPP are very similar to random trees, contrasting dramatically with trees associated with the $p$ spin-glass and random energy models. We also examine critically a recent conjecture on the equivalence between the NPP and a truncated random energy model.
\end{abstract}

\section{INTRODUCTION}

The relevance of the concepts and techniques of statistical physics to understanding the typical behavior of classes of optimization or decision problems had been pointed out by many authors already in the middle of the 1980s (see, e.g., Refs. [1, 2]). However, it was only about ten years later, owing mainly to the finding of a ubiquitous peak in computational cost signaling a transition between easy and difficult instances of optimization problems, that the physics approach has succeeded to attract the attention of the computer science community (see, e.g., Refs. [3-7]). In particular, instances in the phase transition region are now routinely used to benchmark algorithms and search heuristics, and so a precise location of the critical point in addition to the estimate of the width of the transition region has gained considerable practical importance.

The specific optimization problem we consider here is the number partitioning problem (NPP), which is one of the basic NP-complete problems that form the core of the theory of NP-completeness [8]. NPP has an easy formulation: Given $N$ not necessarily distinct positive numbers $a_{1}, \ldots, a_{N}$ find a subset $\mathfrak{X} \subset\{1, \ldots, N\}$ such that

$$
E(\mathfrak{X})=\left|\sum_{j \in \mathfrak{X}} a_{j}-\sum_{j \notin \mathfrak{X}} a_{j}\right|
$$

is minimized. We remark that NPP can be regarded as a Mattis-like Ising spin model with Hamiltonian

$$
H(\mathfrak{X})=E^{2}(\mathfrak{X})=\sum_{i, j} a_{i} a_{j} \sigma_{i} \sigma_{j}
$$

where $\sigma_{i}=+1$ if $i \in \mathfrak{X}$ and $\sigma_{i}=-1$ if $i \notin \mathfrak{X}$ [9]. It is therefore natural to consider $E$ as an energy (cost) landscape over the hypercube; in other words, single spin flips are a natural way of defining a neighborhood relation for the NPP. For concreteness, we will assume from here on that the $a_{i}$ s are independent, identically distributed random variables that take on integer values between 1 and $l$ with equal probability.

A partition $\mathfrak{P}$ is perfect if $E(\mathfrak{P})=0$ or 1 for $\sum_{i} a_{i}$ even or odd, respectively. The existence of perfect partitions depends on the accuracy to which the numbers $a_{i}$ are determined as well as on the size of the problem $N$. The crucial control parameter here is the ratio between the number of bits to which $a_{j}$ is specified and the problem size

$$
\kappa=\frac{\log _{2} l}{N}
$$

The relevance of $\kappa$ can be appreciated by considering the annealed estimate of the expected number of perfect partitions, $\mathcal{S}=2^{N} / l$, so that $\kappa=1-\left(\log _{2} \mathcal{S}\right) / N[10]$. Hence the annealed theory indicates that for $\kappa<1$ there is an asymptotically exponential number of perfect partitions, while for $\kappa>1$ the probability of finding a perfect partition is exponentially small. Extensive numerical simulations and statistical mechanics calculations corroborate the value $\kappa_{c}=1$ as the threshold separating the easy-tosolve from the hard-to-solve regimes $[10,11]$. 
Probabilistic and statistical mechanics analyses of the ground states of the Hamiltonian (2) in the limit of infinite precision $l \rightarrow \infty$, in which the $a_{i}$ s can be viewed as continuous variables, have of course failed to detect the phase transition (see, e.g., Refs. $[9,12,13])$. The thermodynamics for general $l$ was 'solved' under the assumption of self-averaging of the random variables $a_{i}$, but that solution is not completely satisfactory since it predicts a negative entropy for $\kappa>\kappa_{c}$ [11]. Thus a reliable theory for the ground states of the NPP, probably based on the replica method, has yet to be obtained.

Generally, the aforementioned phase transition is defined as a transition from a region in the space of control parameters in which almost all instances have many solutions to a region in which almost all instances have no solution $[3,4]$. Hence, the investigations have relied mainly on exhaustive search procedures, such as branchand-bound algorithms, that guarantee the finding of the global optima, or on statistical mechanics calculations of the expected properties, such as the entropy, of the ground states. In this contribution we seek evidence of this easy/hard transition in the structure of the cost landscape of the optimization problem, focusing on the distribution of optima and on the distribution of cost barriers between these optima. To this end, we code the information about the paths of minimal cost leading to different optima in a tree structure, termed the barrier tree of the cost landscape. The leaves of this tree represent the local optima and the internal nodes the lowest-cost saddles connecting those optima. Barrier trees have been widely used to study protein [14-16], RNA [17, 18], and spinglass [19-22] landscapes.

We find that the structure of the landscape, as measured by the local minima and their connecting saddle points, shows surprisingly little difference in the easy and hard regimes. The sharp transition between these two regimes is revealed only by the difficulty of the landscape, a parameter measuring the maximum ratio of energy barrier to energy gain for the escape from a metastable state, which is directly related to the optimal speed of convergence of simulated annealing [23-27]. We stress that our goal here is not to locate the transition point, which can be achieved by simply looking at the value of the global energy minimum in an ensemble of randomly generated instances (in this sense, a barrier tree always contains the information on whether the given instance is easy or hard), but to seek evidences of the easy and hard regime on other global statistical properties of the energy landscape. Furthermore, we examine a remarkable conjecture about the equivalence between the infinite accuracy version of the NPP and the random cost problem [28], and show that, despite the equivalence at the level of the energy distributions, their barrier trees are completely different.

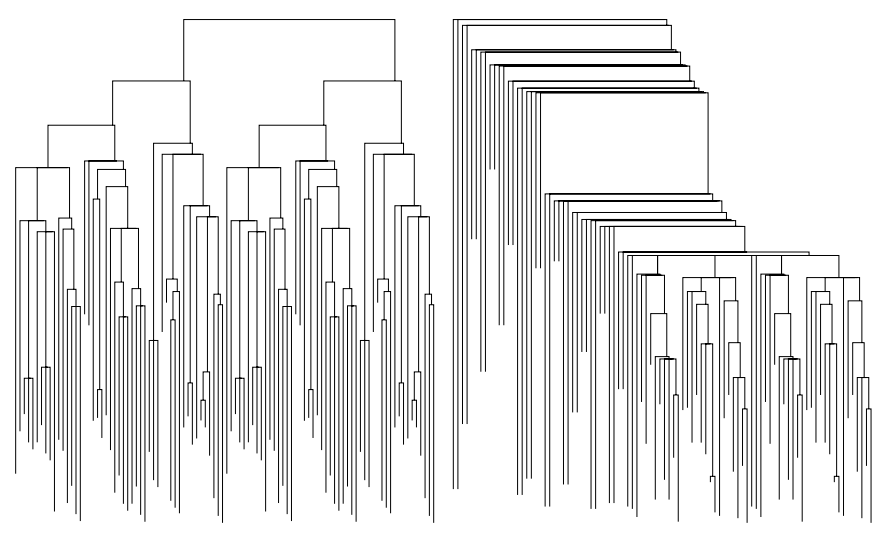

(a)

(b)

FIG. 1: Typical barrier trees for (a) the number partitioning problem with infinite precision numbers and (b) the truncated random energy model (random cost problem) for $N=10$.

\section{BARRIER TREES}

The energy of the lowest saddle point separating two local minima $x$ and $y$ is

$$
E[x, y]=\min _{\mathbf{p} \in \mathbb{P}_{x y}} \max _{z \in \mathbf{p}} E(z)
$$

where $\mathbb{P}_{x y}$ is the set of all paths $\mathbf{p}$ connecting $x$ and $y$ by a series of subsequent spin-flips [19, 29]. The barrier height $B(x)$ of a metastable state $x$ is the minimum height of a saddle point that connects it with metastable state $y$ with strictly smaller energy $E(y)<E(x)$. In symbols,

$$
B(x)=\min \{E[x, y]-E(x) \mid y: E(y)<E(x)\} .
$$

Since a direct evaluation of eq.(4) would require the explicit constructions of all possible paths it does not provide a feasible algorithm for determining $E[x, y]$ even if $N$ is small enough to allow an exhaustive survey of the landscape. The values of $E[x, y]$ and $B(x)$ can, however, be retrieved from the barrier tree of the landscape. The algorithm for constructing these barrier trees is presented in $[17,18]$ (see also Ref. [20] for a detailed account of the algorithm in the spin-glass context). It is implemented in the barriers program [35], which constructs the tree from a sorted list of energy values of all spin configurations in the landscape. In a barrier tree, the leaves of the tree represent the local minima, and the internal nodes represent the saddles, with the barrier sizes given by the length of the branches connecting the local minima to their corresponding saddles. Fig. 1 illustrates typical barrier trees for the problems considered in this paper.

\section{DEPTH AND DIFFICULTY}

The depth $D$ and difficulty $\psi$ of a landscape are measures of the landscape structure that are directly related 
to the performance of simulated annealing [23-27]. The depth of a landscape is defined as the maximum barrier height, $D=\max B(x)$, where the maximum is taken over non-global minima only. In particular, it can be shown that simulated annealing converges almost surely to a ground state if and only if the cooling schedule $T_{k}$ satisfies $\sum_{k \geq 0} \exp \left(-D / T_{k}\right)=\infty$ [23]. The difficulty of the landscape is a dimensionless quantity defined as

$$
\psi=\max \left\{\frac{B(x)}{E(x)-E(\min )}\right\}
$$

where $E(\min )$ is the global energy minimum and, as before, the maximum is taken over local minima only. The difficulty $\psi$ is directly related to the optimal speed of convergence of simulated annealing. It is more convenient to work with the scaled quantity

$$
\lambda=\log _{2}\left(\psi / 2^{N}\right)=\log _{2} \psi-N
$$

instead.

We turn now to the evaluation of the effects on the depth and difficulty measures of a change in the accuracy of the $a_{i}$ s for a fixed problem size. We find that, similarly to many other tree measures discussed in the next section, the depth measure $D$ is independent of the accuracy of the $a_{i}$ s. The effects on the difficulty measure, on the other hand, are striking. Explicitly, in Fig. 2, where each symbol represents the result of the average over 100 landscapes, we show that there is a scaling relation between the average difficulty and the accuracy of number representation: $\langle\lambda\rangle$ converges to a unique function of $(\kappa-1) N$ for large $N$. This scaling function increases linearly for $\kappa<1$ and approaches a constant value of about -2.0 for $\kappa>1$. Hence $\langle\lambda\rangle$ viewed as a function of $\kappa-1$ exhibits a singularity at $\kappa=1$ since it increases linearly with a slope proportional to $N$ as long as $\kappa<1$ and tends towards a constant value for $\kappa>1$. Therefore the rescaled difficulty reflects the phase transition reported in previous analyses of the NPP, which have focused on the singular behavior of the probability of a perfect partition $[10,11]$.

A simple annealed-like argument to explain the behavior of the difficulty depicted in Fig. 2 goes as follows. In the easy regime we have more partitions (spin configurations) than different combinations of numbers, thus the global optimum will probably be a perfect partition, i.e., $E_{\text {min }}=0$ or 1 , while the lowest metastable state will have $E=2$ or 3 . The height of the barrier separating them, however, is essentially the energy of a random configuration, i.e., $B(x)=\mathcal{O}(l)$, and the maximum barrier height will be among the largest numbers in the system, i.e., $\log _{2} \psi \approx \log _{2} l$. Hence subtracting $N$ from both sides yields $\lambda \approx(\kappa-1) N$ in the easy regime. In the hard regime we can only hope to cancel the leading $N$ bits in the optimal configuration, thus we expect a ground-state energy $E_{\min } \approx l / 2^{N}$, while the maximum barrier height is again $\mathcal{O}(l)$ yielding $\log _{2} \psi \approx N$, which is then independent of $\kappa$. Of course, these crude estimates miss polynomial corrections such as the factor $N^{1 / 2}$ that appears in

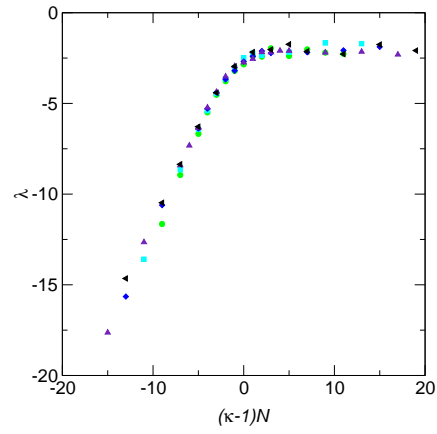

(a)

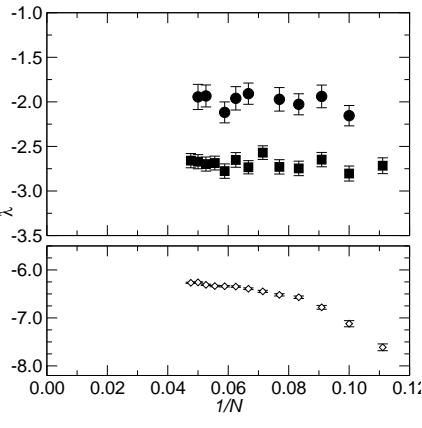

(b)
FIG. 2: (a) Data collapse of the rescaled average logarithmic difficulty $\langle\lambda\rangle$ as function of the rescaled accuracy $(\kappa-1) N$ for problem sizes $N=12,14,16,18$ and 20. (b) Detailed data for $(\kappa-1) N=5(\bullet), 0(\boldsymbol{\square})$, and $-5(\diamond)$ confirm the existence of the scaling function for large $N$.

the rigorous computation of the ground-state energy [12]. Note, however, that we are considering $\log _{2} E_{\min }$, i.e., we ignore only logarithmic corrections which we would expect to arise for more careful estimates of $\log _{2} B(x)$ as well. As the values of $\ln \psi$ vary significantly among different landscapes with the same values of $l$ and $N$ it is not possible to obtain sufficiently accurate estimates of $\langle\lambda\rangle$ that would reveal such corrections unambiguously.

\section{MEASURES OF BARRIER TREE SHAPES}

Since a barrier tree embodies all the relevant quantitative information about the multi-valley structure of an energy landscape, it seems natural to ask if there is any trace of the easy/hard phase transition in the shape of the barrier trees of the NPP landscapes.

Before introducing the standard measures of tree shapes we will consider the effect of the accuracy $l$ on probably the main characteristic of a tree, namely, its number of leaves or minima $n$. In fact, it is tempting to associate the difficulty of a problem with the number of local minima (traps) in its energy landscape representation. Surprisingly, however, we find that the average fraction of local minima does not change with $l$ as soon as $l>N$, i.e., as soon as it becomes unlikely that adjacent configurations have the same energy, see Fig. 3. For very small values of $l$ the number of local optima depends strongly on how degenerate neighbors are treated (data not shown). The important point here is that the fraction of minima stays constant across the easy/hard transition and, in particular, it is given by the formula

$$
\langle n\rangle / 2^{N}=\sqrt{24 / \pi} N^{-3 / 2},
$$

which was obtained in the limit of infinite accuracy [13].

Now we consider five measures of tree shape that were originally used to study phylogenetic trees (see, e.g., Refs. 


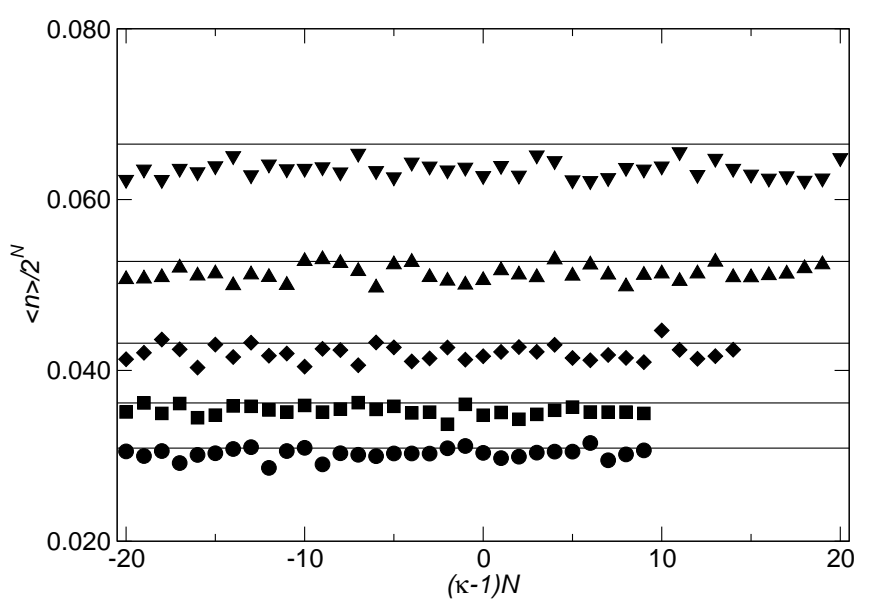

FIG. 3: Average fraction of local minima $\langle n\rangle / 2^{N}$ as a function of the rescaled accuracy $(\kappa-1) N$ for (top to bottom) $N=12,14,16,18$ and 20. Each symbol is the result of an average over 200 landscapes and the horizontal lines are the theoretical predictions for the limit $l \rightarrow \infty$.

$[30,31])$ for the barrier trees resulting from NPP landscapes. These measures provide statistical information about the shape of the barrier tree, mainly its symmetry or balance, and ignore the branch lengths, i.e., the height of the barriers between minima, which were the object of the depth and difficult measures. Recently, we showed that these measures are capable of distinguishing between $p$-spin models with different values of $p[22]$.

Let $d(i, j)$ be the graph-theoretical distance between two nodes of the tree, i.e., the number of edges along the path that connects them. Furthermore, we denote the root of the tree by $\varnothing$. The height of a leaf $k$ is $h_{k}=d(\varnothing, k)$. Equivalently, $h_{k}$ is the number of internal nodes between leaf $k$ and the root $\varnothing$ (inclusive). For each interior node $i$ we have two subtrees with $r_{i}$ and $s_{i}$ leaves, respectively. We assume $r_{i} \geq s_{i}$. The subtreeheight of an interior node $i$ is $m_{i}=\max _{k \in T_{i}} d(i, k)$ where the maximum is taken over all leaves $k$ in the subtree $T_{i}$ below $i$, i.e., the subtree of which $i$ is the root.

With this notation we may define the following five characteristic values for the shape of a binary rooted tree:

1. $H=\frac{1}{n} \sum_{k=1}^{n} h_{k}$ is the average height of a leaf in the tree.

2. $\sigma_{H}=\sqrt{\frac{1}{n} \sum_{k=1}^{n}\left(h_{k}-H\right)^{2}}$, is the standard deviation of the leaf height.

3. $C=\frac{2}{n(n-3)+2} \sum_{i=1}^{n-1}\left(r_{i}-s_{i}\right)$ is a measure for the imbalance of trees with $n>2$.

4. $B_{1}=\sum_{i \neq \varnothing} 1 / m_{i}$ is the average inverse subtree height, where the sum is taken over all $n-2$ internal nodes $i$ excluding the root $\varnothing$.

5. $B_{2}=\sum_{k=1}^{n} 2^{-h_{k}} h_{k}$ is an alternatively weighted average leaf-height.

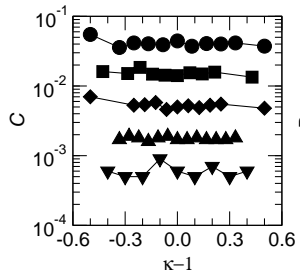

(a)

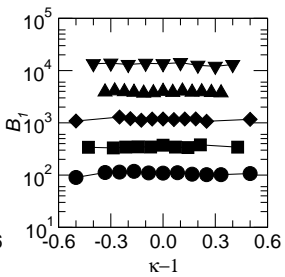

(b)

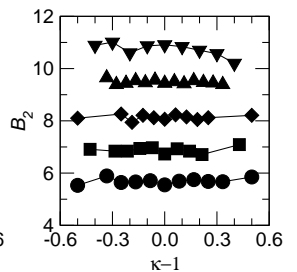

(c)
FIG. 4: None of the measures of the tree-shape shown here reflect the easy/hard transition of the $\operatorname{NPP}$ (a) imblance $C$, (b) inverse subtree height $B_{1}$, (c) weighted leaf-height $B_{2}$. The data for $N=12(\boldsymbol{\nabla}), 14(\boldsymbol{\Delta}), 16(\bullet), 18(\boldsymbol{\square})$, and $20(\bullet)$ are averages over 100 landscapes.

The physical meaning of $H$ and $\sigma_{H}$ is clear. We mention only that for random trees (e.g., trees produced by the neutral genealogical process [32]) the expected value of $H$ increases as $\ln n$. In addition, $\sigma_{H}=0$ for a completely symmetric tree. The imbalance measure $C$ assigns a weight proportional to the the number of leaves to each one of the two subtrees branching out an internal node. These weight differences are then averaged and normalized over all internal nodes of the tree. The value of $C$ increases from 0 for a completely symmetric tree to 1 for a completely asymmetric tree. The statistic $B_{1}$ looks at the longest possible path $m_{i}$ from each internal node $i$ to any of the leaves in its subtree. The statistic $B_{2}$ is based on an index of information content. For highly asymmetric trees, such as those produced by $p$-spin landscapes [22], it will quickly converge to the value $B_{2}=2$. Both $B_{1}$ and $B_{2}$ have smaller values for increasingly asymmetric trees.

Somewhat surprisingly, neither of these measures exhibits a non-trivial dependence on $\kappa$, as shown in Fig. 4 for the measures $C, B_{1}$ and $B_{2}$. The other quantities $H$ and $\sigma_{H}$ behave analogously but have a larger scatter for large $N$. Therefore it is impossible to tell the easy from the hard regime by visual inspection of the barrier tree or by simply measuring branch lengths, as done in the depth measure. The only effective measure involves a nontrivial balance between branch lengths and leaf energies.

\section{TRUNCATED RANDOM ENERGY MODEL}

A very interesting though poorly explored finding concerns the equivalence between the NPP in the limit of infinite precision, where the $a_{i}$ become real random variables distributed uniformly in the unit interval, and the symmetrized truncated random energy model (REM) or random cost problem [28]. In particular, for large problem sizes the energies of two or more distinct configurations of the NPP become statistically independent and the $M=2^{N-1}$ distinct energies values for unconstrained 
partitions are distributed according to

$$
P(E)=\frac{2}{\sqrt{2 \pi \mu_{\infty} N}} \exp \left(-\frac{E^{2}}{2 \mu_{\infty} N}\right) \Theta(E)
$$

where $\mu_{\infty}=\left\langle a^{2}\right\rangle=1 / 3$ is the second moment of the $a_{j}$ in the corresponding number partitioning problem. Hence the bold claim that the NPP is essentially equivalent to a truncated REM [28]. The main application of Eq. (9) is the derivation of the probability density of the minimum energy $E_{\min }$ using trite arguments of extreme statistics [28],

$$
\rho\left(E_{\min }\right)=M P(0) \exp \left[-M P(0) E_{\min }\right],
$$

from which the expected minimum energy follows trivially,

$$
\left\langle E_{\min }\right\rangle=\sqrt{2 \pi / 3} N^{-1 / 2} 2^{-N}
$$

in agreement with the known numerical [13] and analytical [11] results for the NPP.

However, in order for the equivalence at the level of the energy distribution between the NPP and the truncated REM to have any use in guiding the design of search heuristics for the NPP, it is important that other features of the two problems, such as their multi-valley structures, are similar too. In fact, a glance at Fig. 1 is already sufficient to reveal the deep structural difference between the barrier trees of these problems, and the remainder of this section is aimed at quantifying these differences.

The expected number of minima can be easily calculated for any random energy model with finite probability density over the reals [33, 34]. The argument goes as follows. Fix an arbitrary spin configuration $\sigma=\left(\sigma_{1}, \sigma_{2}, \ldots, \sigma_{N}\right)$ and consider all its $\mathrm{N}$ neighbors. Since we assign to each spin configuration a random energy value drawn from the continuous distribution (9) we conclude that (i) all these $N+1$ energies are distinct with probability 1 ; and (ii) $E(\sigma)$ is the smallest of the $\mathrm{N}+1$ numbers with a probability of $1 /(N+1)$. Hence $\sigma$ is a local minimum with probability $1 /(N+1)$ and so the fraction of local minima is

$$
\langle n\rangle / 2^{N}=1 /(N+1)
$$

which, for large $N$, is larger than the result for the NPP [see Eq.(8)] by a factor of order of $N^{1 / 2}$.

In Fig. 5 we present the tree size $(n)$ dependence of the barrier tree measures $C$ and $H$ for the NPP and the symmetrized truncated REM. A useful standard here, also shown in this figure, is the random trees, generated as follows. First, create $n$ nodes (the leaves) and put them in a set $A$. Next, remove two random nodes $x$ and $y$ from $A$, create a new node $z$ and make $x$ and $y$ its two children, and put $z$ in the set $A$. Repeat this procedure until there is only one node left in $A$, which will be the root of the tree. Random trees are important from the biological viewpoint because they arise from the neutral genealogical process [32]. The symmetrized truncated
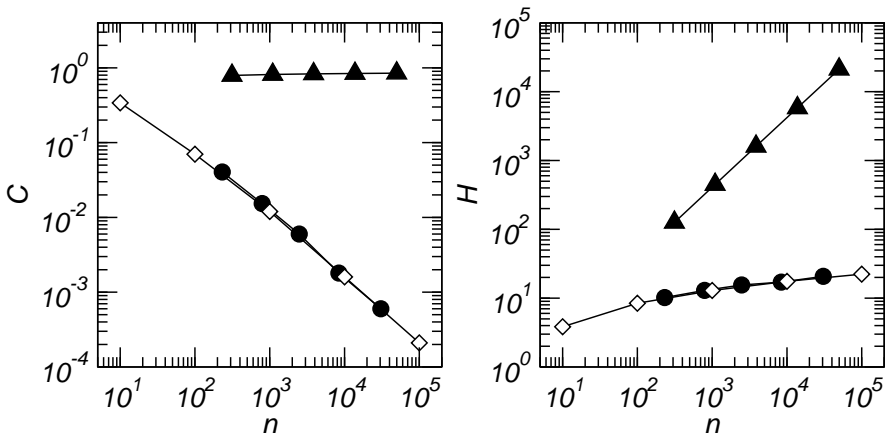

FIG. 5: Tree balance measures $C$ and $H$ as functions of the tree size $n$ for the symmetrized truncated REM $(\mathbf{\Lambda})$, the NPP $(\bullet)$ and random trees $(\diamond)$. The solid lines are numerical fittings: $H=0.4 n$ and $C=0.6+0.1 \ln \ln (n)$ for the REM, and $H=-1+2 \ln (n)$ and $C=5 / n$ for the NPP and random trees.

REM presents the same scaling on $n$ as the $p$-spin models, namely, $H \sim n$ and $C \sim \ln \ln (n)$ within the range of $n$ considered [22]. Of course, since $C \in[0,1]$ this scaling cannot be valid for $n \rightarrow \infty$, but the double logarithmic dependence guarantees its validity for a very large range of tree sizes. Actually, as far as the five statistics introduced in Sec. IV are concerned, there is no significative differences between the symmetrized truncated REM and the standard REM, both models producing then extremely unbalanced trees. These measures, however, differ dramatically between the NPP and all previous spin-glass models analysed [22]. Surprisingly, the NPP barrier trees are practically indistinguishable from random trees and, in particular, the tree measures obey the same scaling relation with the tree size, $H \sim \ln (n)$ and $C \sim 1 / n$. Hence these trees, in stark contrast with the $p$-spin model and REM barrier trees, become more and more balanced as $n$ increases.

Since the equivalence between NPP and the truncated REM discussed above was proved only in the case of infinite precision numbers (i.e., $\kappa \rightarrow \infty$ ) it provides no clues about the easy/hard phase transition that takes place at $\kappa=1$. Nevertheless, it is natural to ask if the integer counterpart of the truncated REM, obtained by considering the integer-valued energies $E^{\prime}=\lfloor E\rfloor$ where $E$ is drawn from the distribution (9) with $\mu_{\infty}$ replaced by $\mu_{l}=l(l+1)(2 l+1) / 6$, exhibits a phase transition. This can easily be answered by calculating the probability that a partition is perfect (i.e., $E_{\min }^{\prime}=0$ or 1 ) which is given by

$$
P_{\text {perf }}=\int_{0}^{2} d E_{\min } \rho\left(E_{\min }\right)=1-\exp (-2 \xi)
$$

where $\xi=2^{N} /\left(2 \pi \mu_{l} N\right)^{1 / 2}$. Although one clearly recovers the easy $\left(P_{\text {perf }} \approx 1\right)$ and the hard $\left(P_{\text {perf }} \approx 0\right)$ regimes depending on whether the ratio $\left(\log _{2} l\right) / N$ is very small or very large, respectively, there is definitely no phase transition separating them. 


\section{CONCLUSION}

Phase transitions in physical systems are characterized by the appearance of singularities in some observables, known as the order parameters of the system, such as, e.g., the gas density in the boiling transition. In the case of mean-field spin-glass models the order parameter directly reflects the hierarchical organization of pure states in a complex multi-valley structure [2]. Therefore one expects that some features of that structure must undergo abrupt changes when the critical point is approached. Unfortunately, the vast majority of the phase transitions take place at finite temperature, while a direct study of the landscape properties of spin-glass models based on natural quantities, such as saddle points and minima, is feasible at zero temperature only. In that sense, the easy/hard phase transitions in optimization problems [4] in general, and in the number partitioning problem (NPP) $[10,11]$ considered here, provide a unique chance to study how the onset of the phase transition affects the organization of the metastable states of a disordered spin system.

Somewhat surprisingly, we find that almost all features of the landscape, which we have properly mapped into a tree structure through the barriers program, are insensitive to the onset of the easy/hard phase transition that takes place when the number of bits needed to specify a number $a_{i}$ equals the problem size, i.e., $\kappa=\left(\log _{2} l\right) / N=1$. Interestingly, only one of the measures studied, premonitorily termed difficulty in the mathematical literature of simulated annealing [24], exhibits a singular behavior at the critical point. As a result, the quality of the optima found by simulated anneal- ing will probably depend strongly on whether the control parameters set the instance in the easy $(\kappa<1)$ or hard $(\kappa>1)$ regime.

An important by-product of our study of the NPP landscape is the finding that the resulting barrier trees are very similar to random trees, and so they become completely balanced (symmetric) in the limit of large system sizes $N$ or, equivalently, large tree sizes $n$. These trees contrast drastically with the barriers trees resulting from the $p$ spin-glass, the random energy or the symmetrized truncated random energy landscapes, which become completely unbalanced in that limit. In this context, we note that although there is an equivalence at the level of the energy distribution between the NPP and the truncated random energy model, the statistical properties of their energy landscapes are very different, and probably so are the performances of local search heuristics in finding near-global solutions to these problems. Actually, the similarity of the NPP barrier trees with random trees may be part of the explanation for the failure of local search techniques to produce good solutions to this optimization problem.

\section{Acknowledgments}

Thanks to Christoph Flamm at the University of Vienna, Austria, for his help with the barriers program. This research was supported by Fundação de Amparo à Pesquisa do Estado de São Paulo (FAPESP), project 99/09644-9. The work of J.F.F. is supported in part by $\mathrm{CNPq}$ and $\mathrm{WH}$ is supported by FAPESP. P.F.S. gratefully acknowledges the hospitality of the Instituto de Física de São Carlos in Nov. 2002.
[1] P. W. Anderson, Physica A 140, 405 (1986).

[2] M. Mézard, G. Parisi and M. A. Virasoro, Spin Glass Theory and Beyond (World Scientific, Singapore, 1987).

[3] S. Kirkpatrick and B. Selman, Science 264, 1297 (1994).

[4] T. Hogg, B. A. Huberman and C. P. Williams, Artif. Intell. 81, 1 (1996).

[5] B. Hayes, Am. Sci. 85, 108 (1997).

[6] R. Monasson, R. Zecchina, S. Kirkpatrick, B. Selman and L. Troyansky, Nature 400, 133 (1999).

[7] O. Martin, R. Monasson and R. Zecchina, Theor. Comp. Sci. 265, 3 (2001).

[8] M. R. Garey and D. S. Johnson, Computers and Intractability: A Guide to the Theory of NP-Completeness (Freeman, New York, 1979).

[9] Y. Fu, in Lectures in the Science of Complexity edited by D. L. Stein (Addison-Wesley, Reading, 1989).

[10] I. P. Gent and T. Walsh, Comput. Intell. 14, 430 (1998).

[11] S. Mertens, Phys. Rev. Lett. 81, 4281 (1998).

[12] N. Karmarkar, R. M. Karp, G. S. Lueker, and A. M. Odlyzko, J. Appl. Probab. 23, 626 (1986).

[13] F. F. Ferreira and J. F. Fontanari, J. Phys. A 31, 3417 (1998); Physica A 269, 58 (1999).

[14] O. M. Becker and M. Karplus, J. Chem. Phys. 106, 1495
(1997).

[15] D. J. Wales, M. A. Miller and T. R. Walsh, Nature 394, 758 (1998).

[16] P. Garstecki, T. X. Hoang and M. Cieplak, Phys. Rev. E 60, 3219 (1999).

[17] C. Flamm, W. Fontana, I. L. Hofacker and P. Schuster, RNA 6, 325 (2000).

[18] C. Flamm, I. L. Hofacker, P. F. Stadler and M. T. Wolfinger, Z. Phys. Chem. 216, 155 (2002).

[19] K. Nemoto, J. Phys. A 21, L287 (1988).

[20] F. F. Ferreira, J. F. Fontanari and P. F. Stadler, J. Phys. A 33, 8635 (2000).

[21] J. F. Fontanari and P. F. Stadler, J. Phys. A 35, 1509 (2002).

[22] W. Hordijk, J. F. Fontanari, and P. F. Stadler, e-print cond-mat/0302041.

[23] B. Hajek, Math. Operations Res. 13, 311 (1988).

[24] O. Catoni, Ann. Probab. 20, 1109 (1992).

[25] W. Kern, Discr. Appl. Math. 43, 115 (1993)

[26] J. Ryan, Discr. Appl. Math. 56, 75 (1995).

[27] O. Catoni, Lecture Notes in Mathematics 709, 69 (1999).

[28] S. Mertens, Phys. Rev. Lett. 84, 1347 (2000).

[29] A. M. Vertechi and M. A. Virasoro, J. Phys. France 50, 
2325 (1989).

[30] K-T. Shao and R. R. Sokal, Syst. Zool. 39, 266 (1990).

[31] M. Kirkpatrick and M. Slatkin, Evolution 47, 1171 (1993).

[32] J. Felsenstein, Inferring Phylogenies (Sinauer Associates, Sunderland, 2002).
[33] B. Derrida, Phys. Rev. B 24, 2613 (1981).

[34] D. J. Gross and M. Mézard, Nucl. Phys. B 240, 431 (1984).

[35] The source code is available at http://www.tbi.univie.ac.at/ ivo/RNA/Barriers/. 